\title{
POWER ASSISTANT SYSTEM FOR HEAVY WORKER
}

\author{
Kyoung Taik Park* \\ Center of Robotics \& Mechatronics, Nano System Research Division, Daejeon, Korea \\ * Corresponding author (ktpark@kimm.re.kr)
}

\begin{abstract}
This study presents the power assistance system of working suit type for heavy worker in construction site of steel bridge and high building. The heavy worker carrying several tools such as hammer, grinder, window frame and window glass should be stayed to do handling work in closed working space or inside internal wall of large complex block and in long time. The power suit system is realized by developing the mechanism and control technology to reduce the working load to be transferred to wrist, arm and shoulder of heavy worker. It is developed to realize the human friendly optimal working motion. This study shows the link design of multi-axis mechanism to do handling work of a variety of motion. It shows the control technology of handling work system to reduce the effect of the weight of handling tool and wearable suit system and the working load of handling process such as the reaction force of tools.
\end{abstract}

Keywords: Power Assistant Suit, Hydraulic Actuator, Handling Worker, Heavy Worker, Power Control

\section{INTRODUCTION}

In the developed country the field of the handling process in construction site is only directly doing by heavy worker because it is not developed in the automatic process and it has very coarse working environment. Even though in Japan the industries of building construction were tried to develop the automatic process of handling work several times, they did not obtain the remarkable results and they are also still trying to develop the automatic handling process in building construction. In the developed country most of the handling process of the automobile manufacturing company is accomplished in almost perfectly automatic process and the products of the major manufacturing company of materials and equipments are used in most of automobile manufacturing company. In the domestic companies of building construction most of the handling process is still dependent on the operators of handling tools and also in the other developed countries it is still dependent on them. Though some of the domestic companies are tried to develop the automatic process of handling, they did not obtain the considerable results like Japan. The characteristic of the real site of handling process of building construction industry has the difficulty to be changed into the automatic process. It has very coarse environment and the high pressure of working load for heavy workers to work in long time. The heavy worker carrying several tools such as hammer, window frame and window glass should be stayed to do handling work in closed working space or inside internal wall of construction block and in long time. The goal of this study is to help the worker to smoothly do the handling work in required conditions. Specially, by developing the motion mechanism and control technology to reduce the working load to be transferred into the body system of operator the working suit system is developed to realize the optimal and human friendly working motion $[1,2]$.

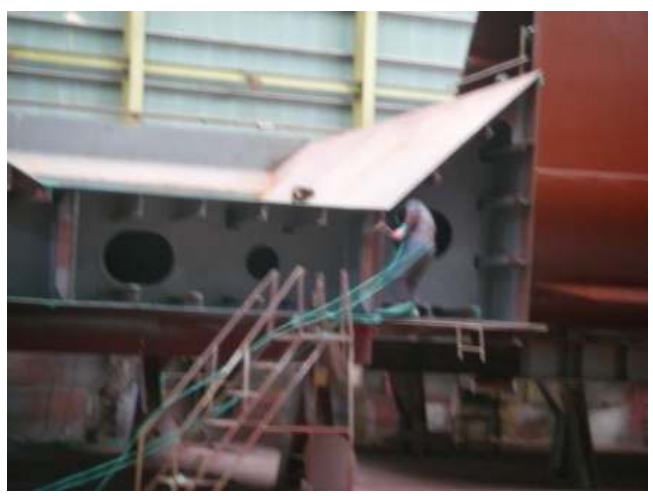

Figure 1. Heavy working in ship building site 
And also this study shows the design of multi-axis link mechanism to smoothly do handling work in a variety of motion. It shows the control technology of working suit system to reduce the effect of the weight of handling tool and wearable suit structure and the working load of handling process such as the reaction force of tools $[3,4,5]$. The working motion to design the mechanism and structure of multi-axis link type to realize the working motion in a variety of motion is analyzed and the human-friendly handling work assistant system is developed by introducing the engineering design concept of human body. The Hframe type of the working suit system is designed and manufactured and the controller to reduce the effect of system weight and working load is designed and manufactured.

\section{HYDRAULIC ACTUATOR FOR POWER ASSISTANT SYSTEM}

\section{A. Required System Function and Performance}

The scope of working motion is expressed in the angle of handling tool motion to move in required conditions with carrying a variety of tools such as hammer, grinder and roller. As this scope becomes larger and it is possible to do the handling work in complex shape and narrow block space. The driving axis of working motion is the number of axis of link type to construct the main axis of working suit system. Generally, the working motion becomes much smoother as the number of this axis becomes larger. The kind of handling tools is generally classified into several categories to do the handling work in different handling method by using different handling tools such as hammer, grinder and roller. The number of driving axis of related motion is defined in relation with arm and wrist motion of worker when the operator in working suit is doing the handling work. The working velocity is defined as the velocity of handling tools to move in working path and it is closely related with the productivity of handling work. The working load is defined as the total force to be pulled and pushed at end of the working suit system or to be hanged on it $[6,7,8]$.

\section{B. Power Assistant Suit System for Heavy Worker}

The working suit system is designed to be used when the high vibration of drilling tool is occurred. The reaction of drilling pressure and self-weight of handling tool are transferred to the shoulder and arm of heavy worker and the accumulated fatigue in body system is occurred. As results, the productivity of handling work is degraded and it is designed to prevent the industrial accident from doing the handling work. The handling assistant suit system is composed in the complex mechanism of multi- axis link type to do handling work in a variety of degree of freedom and it is possible to be wearable and to amplify the articulation power of heavy worker. To do this, the joints of working suit system are rotated by electrical motor or hydraulic actuator. The hydraulic actuators are installed on links between thigh and shin to perform the linear motion. These actuators take charge of the roll to rotate shin links. Like these, if the links are rotated by the actuator to do linear motion the joints have the disadvantage to be interfered by the actuators.

\section{Hydraulic Actuator for Power Assistant Suit System}

In hydraulic system there are several kinds of hydraulic driving motor to make rotating motion. This kind of actuator has not only low efficiency of power but also the difficulty of exact closing motion because of pressure difference and flow leakage between high pressure room and low pressure room in hydraulic actuator system. The new hydraulic cylinder motor is proposed to solve demerits of hydraulic motor and to make rotating motion possible. The hydraulic cylinder motor is the apparatus to convert linear motion into rotating motion by combination of one or more than two pulleys, wire-rope and directional change valve. Figure 2 shows the schematic diagram of apparatus and elements are the followings.

(1) : Hydraulic Power Source, (2): Check Valve in Supply, (3) : Flow Control Valve in Supply, (4): Directional Change Valve, (5) and (11): Single One-Rod Hydraulic Cylinder with Restoration Spring, (6) and (10): Wire Rope Connector, (7): Wire Rope, (8): Follower Pulley, (9): Pulley Hinge, (12) : Flow Control Valve in Restoration Side, (13) : Check Valve in Restoration Side, 14): Flow Reservoir, 
(15) Rope Fixer, (16) : Idle Pulley, (17) : Pulley-Rod Connector, (18) : Driving Pulley, (19): Pulley-Rope Fixer, (20) : Idle Pulley of Angle Change

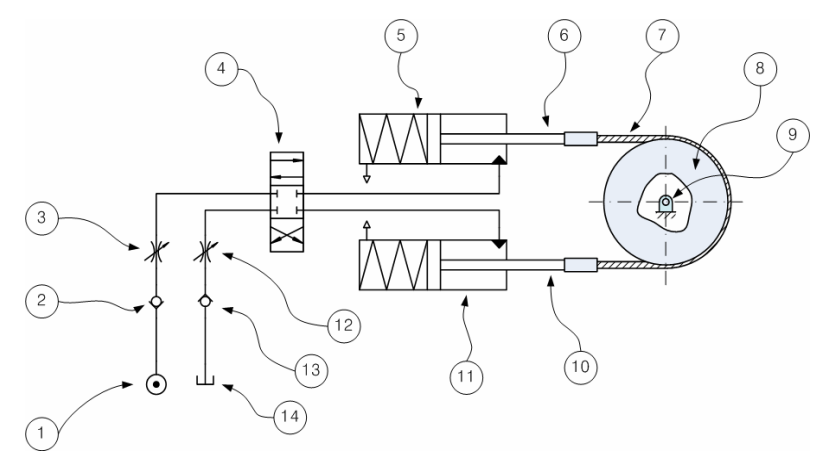

Figure 2. Configuration of hydraulic cylinder motor

The special feature of this system is to realize more than $1 / 2$ rotation motion of idle pulley to be installed on linkjoint parts. And also the special feature of this system has the simple structure and high efficiency of power because of small leakage of operating oil compared with hydraulic motor to use flow power directly. And it has the possibility of exact closing motion because of adjusting of back pressure in internal pressure room and rotation motion in less than or more than half rotation. Figure 3 and 4 show the principle of operation of proposed system that are expressed in clockwise direction and counterclockwise direction of follower pulley. The state of clockwise direction of follower pulley(8) is shown in Figure 3.

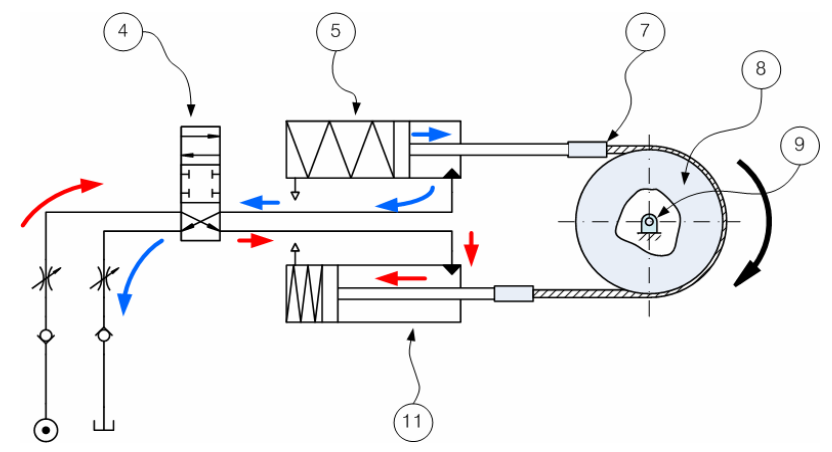

Figure 3. Operation state of follower pulley in clockwise direction

The flow power to be supplied from the flow power supplier(1) is passing through check valve(2) of supplier to be installed to make flow direction steady and to protect backward flow and the high pressure flow to be passing through direction change valve(4) is supplied to hydraulic cylinder(11). The other flow in hydraulic cylinder(5) is passing through flow control valve(12) and flow check valve(13) in restoration(12) and then returned to flow reservoir(14). The rod of hydraulic cylinder(11) is connected to the connector(10) of wire rope and wire rope(7) is wound up the circular face of the follower pulley(8). The wire rope(7) is rotated in direction to be decided by moving direction of hydraulic cylinder(11) and then the follower pulley(8) is coupled with it. At this time, the flow of pressure room of hydraulic cylinder(5) prevents the rapid rotation of the follower pulley(8) by supplying the tension of wire rope(7) and by restraining the restoration flow to be crossed by flow control valve(12). Therefore, the follower pulley(8) is rotated in clockwise direction by forming the back pressure of hydraulic cylinder(5) and linear motion of hydraulic cylinder(11).

Figure 4 shows the state that the follower pulley(8) is rotated in counterclockwise direction. The flow power to be supplied from the flow power supplier(1) is passing through check valve(2) of supplier to be installed to make flow direction steady and to protect backward flow and the high pressure flow to be passing through direction change valve(4) is supplied to hydraulic cylinder(5). The other flow in hydraulic cylinder(11) is passing through flow control valve(12) and flow check valve(13) in restoration(12) and then returned to flow reservoir(14).

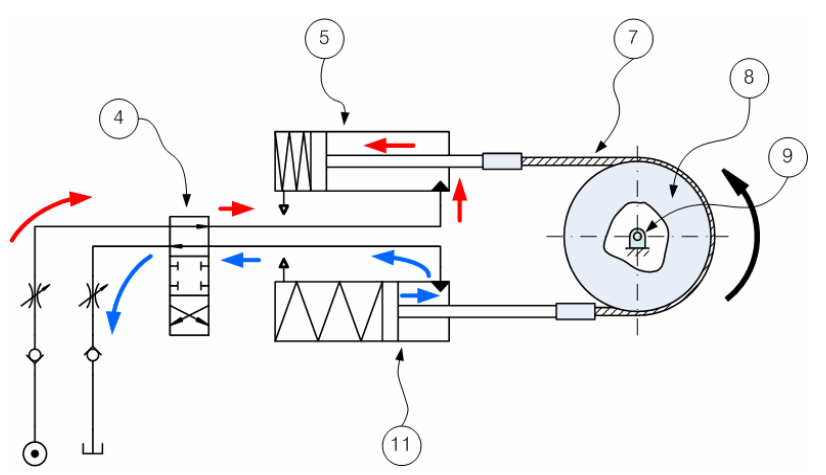

Figure 4. Operation state of follower pulley in counter clockwise direction

The rod of hydraulic cylinder(5) is connected to the connector(6) of wire rope and wire rope(7) is wound up the 
circular face of the follower pulley(8). The wire rope(7) is rotated in direction to be decided by moving direction of hydraulic cylinder(5) and then the follower pulley(8) is coupled with it. At this time, the flow of pressure room of hydraulic cylinder(11) prevents the rapid rotation of the follower pulley(8) by supplying the tension of wire rope(7) and by restraining the restoration flow to be crossed by flow control valve(12). Therefore, the follower pulley(8) is rotated in clockwise direction by forming the back pressure of hydraulic cylinder(11) and linear motion of hydraulic cylinder(5).

Figure 5 shows the configuration of hydraulic cylinder motor and installed idle pulley in another example of Figure 2. If the flow power is supplied to hydraulic cylinder(5), the idle pulley to be connected to the $\operatorname{rod}(17)$ of hydraulic cylinder is rotated in clockwise direction. The wire rope(18) to be connected to rope fixer(15) is moved by idle pulley(16) in right direction. At this time, the wire rope rotate the follower pulley in clockwise direction to be connected by pulley-rope connector(19).

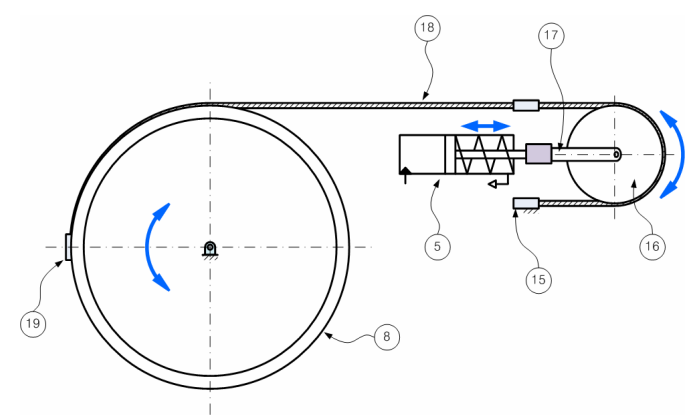

Figure 5. Hydraulic cylinder motor with idle pulley

To rotate the follower pulley in counterclockwise direction the same system configuration of Figure 3 is established on bottom side as shown in Figure 6. Figure 7 shows that the system has the different configuration and the same role of system as shown in Figure 6. The hydraulic cylinder deriving actuator is applied to the arm design of the working habiliment system. The possibility of 2 DOF pitch and roll motion with respect to wrist and elbow is tested in the designed method shown in Figure 6 and 7.

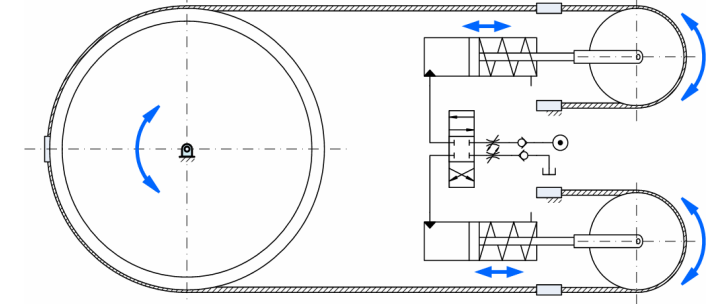

Figure 6. Hydraulic motor(1) with rotation motion in clockwise and counter clockwise direction

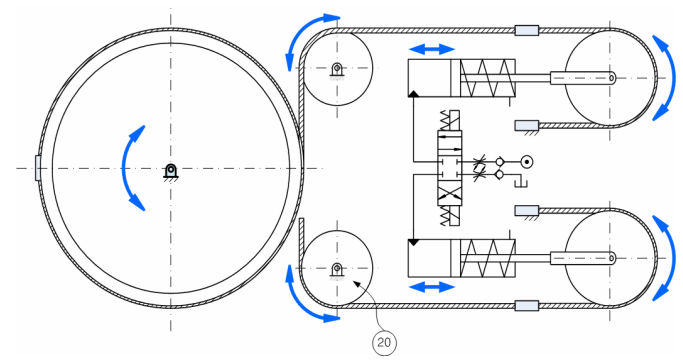

Figure 7. Hydraulic motor(2) with rotation motion in clockwise and counterclockwise direction

The 2 DOF arm part of working habiliment system that wrist and elbow are united in one body is tested by the 3D drawing CAD shown Figure 7 and 8. The results of simulation of modeling of arm part show the exact operation that the interference of actuator structure and links is not consisted. This is should be considered in manufacturing process.

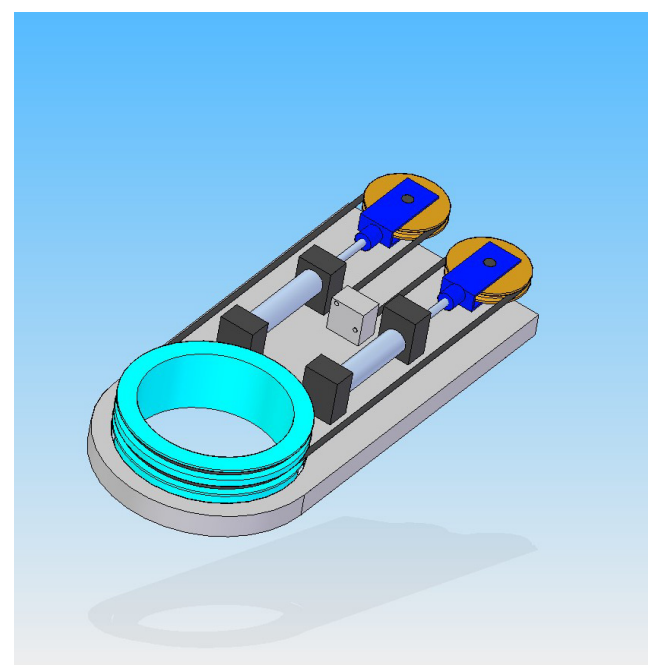

Figure 8. Hydraulic motor with idle pulley

The shoulder joint has 3 DOF mechanism that the additional 1 DOF mechanism is added to mechanism of 2 
DOF upper arm part. The shoulder joint has 3 DOF mechanism that the additional 1 DOF mechanism is added to mechanism of 2 DOF upper arm part.

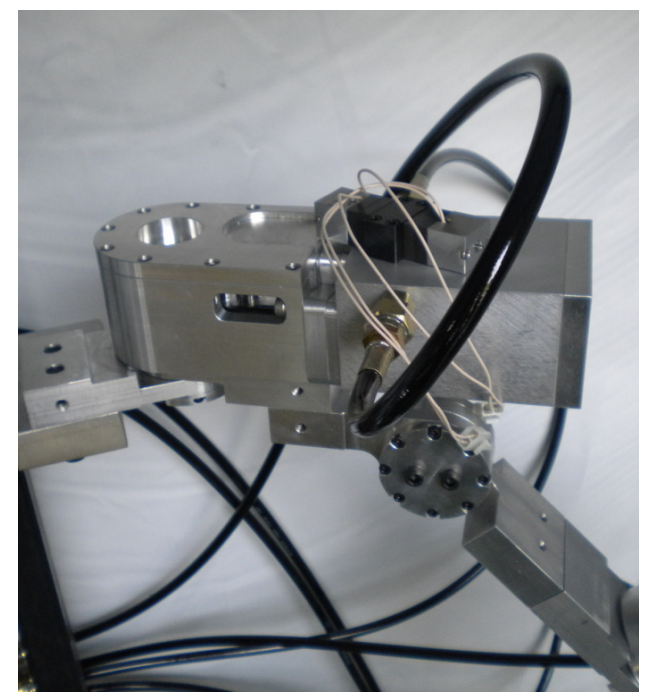

Figure 9. Hydraulic motor installed on joint

The experimental models of actuator, joint and link are manufactured and tested as shown in Figure 9. The power pack supplies hydraulic power to actuator. The hydraulic line, electrical line and valves to control the system motion are shown in Figure 10.

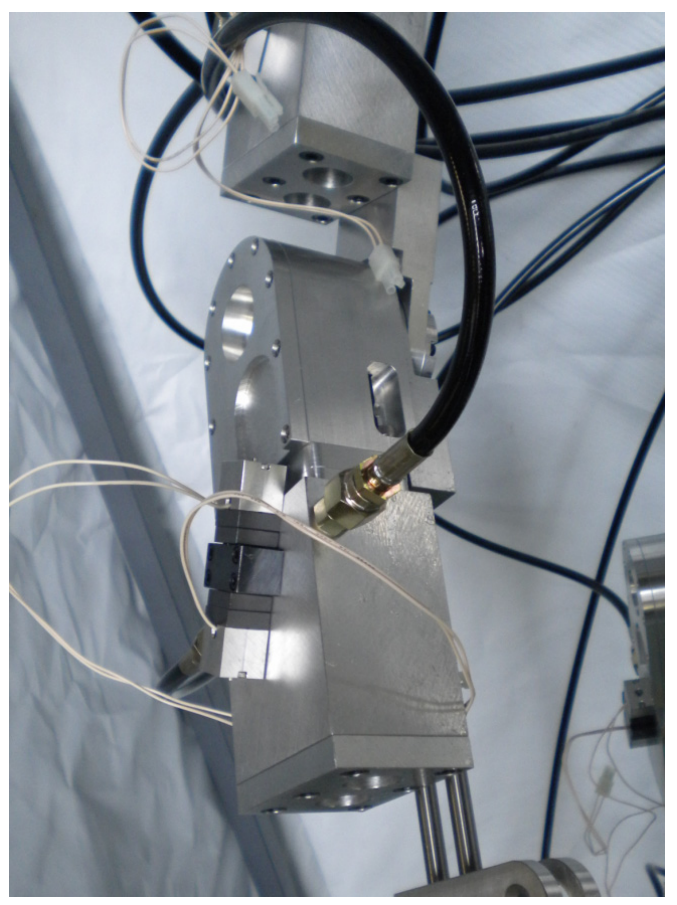

Figure 10. Hydraulic motor installed on joint
The leg part of working suit system requires the fundamental gait motion of human body and the control function to support the upper human body and to maintain the balance of the total human body is required.

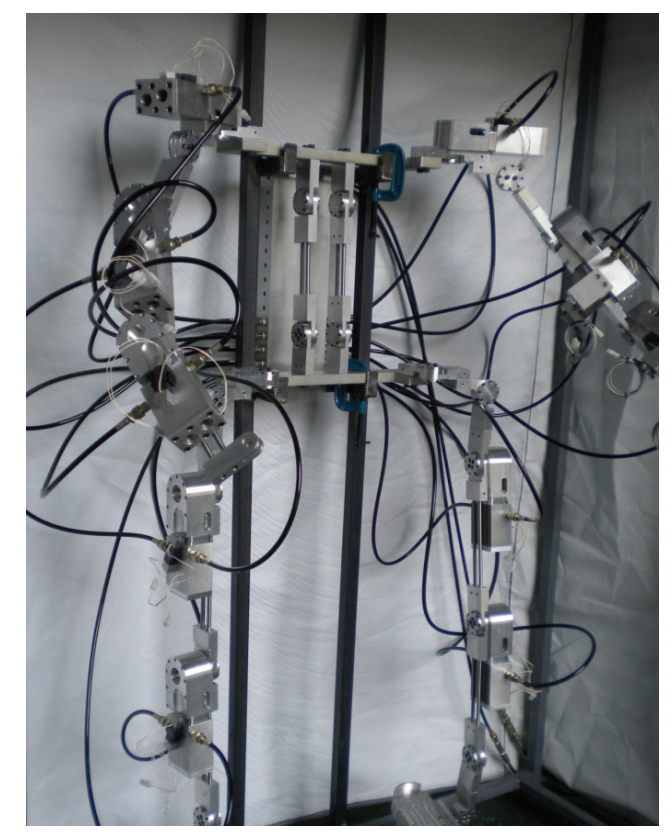

Figure 11. Power assistant system for heavy worker

\section{COCLUSION}

The first function of the leg part is to realize the fundamental characteristics of the gait motion. To perform the smooth gait motion as like human body the leg part should require a variety of degree of freedom of hip, knee, ankle, shoulder, elbow and wrist joint motion. The skeleton structure of working suit system to be assembled with links and joints shows in Figure 10. The size of working suit system is suitable to be wearable for heavy worker. The hydraulic actuators and control valves are installed on the proto type of skeleton structure. To improve the productivity of ship building industry the repeated and accumulated fatigue of heavy worker should be reduced in manufacturing process of small and large size of block to be assembled in dock site. In this study the multi-axis link mechanism to smoothly do painting motion in a variety of working path is designed and manufactured. The structures of hydraulic actuator and joint are designed and manufactured to be wearable for heavy worker. 
In near future the mechanism and control technology of working suit system to reduce the effect of the weight of painting tool and wearable structure and the working load of painting process such as the reaction force of spray gun will be executed.

\section{ACKNOWLEDGEMENT}

This work is supported by the Technology Innovation Program funded by the Ministry of Knowledge Economy(MKE, Korea)

\section{REFERENCES}

[1] A. Schiele, "Undesired Constraint Forces in nonergonomic wearable Exoskeletons," Extended Abstract for IROS'07 Workshop on Assistive Technologies: Rehabilitation and Assistive Robotics.

[2] Joel C. Perry, Jacob Rosen, "Design of a 7 Degree-ofFreedom Upper-Limb Powered Exoskeleton," BioRobo 2006-The first IEEE/RAS-EMBS International Conference on Biomedical Robotics and Biomechatronics Pisa, Tuscany, Italy, February 20-22, 2006.

[3] N. Hogan, "Impedance control, an approach to manipulation: Part I-III", ASME Journal of Dynamics Systems, Measurement, and Control, Vol. 107, 1985, pp. 124.

[4] T. Yoshikawa and X. Zheng, "Coordinated dynamics hybrid position/force control for multiple robot manipulators handing one constrained object," International Journal of Robotics Research, Vol. 12, No. 3, pp. 219-230, 1993.

[5] L. Huang, S. S. Ge, and T. H. Lee, "An Adaptive Impedance Control Scheme for Constrained Robots," International Journal of Computers, Systems and Signals, Vol. 5(2), pp. 17-26, 2004.

[6] C. H. Park and K. T. Park, "Design and Kinematics Analysis of Dual Arm Robot Manipulator for Precision Assembly,” INDIN2008 IEEE, Daejeon, Korea, 2008.

[7] K. T. Park and C. T. Park, "Performance Evaluation of Industrial Dual-Arm Robot," ICSMA2008 ICROS, Seoul, Korea, 2008.

[8] Russdon Angold, Nathan Harding and Homayoon
Kazerooni, Semi-Powered Lower Extremity Exoskeleton, International Application Number : PCT/US2006/014227, International Publication Number: WO 2006/113520 A2. 